\title{
A viable Starobinsky-like inflationary scenario in the light of Planck and BICEP2 results ${ }^{1}$
}

\author{
Spyros Basilakos ${ }^{a}$, José Ademir Sales Lima $^{b}$, Joan Solà ${ }^{c}$ \\ ${ }^{a}$ Academy of Athens, Research Center for Astronomy and Applied Mathematics, \\ Soranou Efesiou 4, 11527, Athens, Greece, e-mail: svasil@Academyofathens.gr \\ ${ }^{b}$ Departamento de Astronomia, Universidade de São Paulo, Rua do Matão 1226, \\ 05508-900, São Paulo, SP, Brazil, e-mail: jas.lima@iag.usp.br \\ ${ }^{c}$ High Energy Physics Group, Departament d'Estructura i Constituents de la \\ Matèria, and Institut de Ciències del Cosmos, Universitat de Barcelona, \\ Av. Diagonal 647 E-08028 Barcelona, Catalonia, Spain, e-mail: sola@ecm.ub.edu
}

(Submission date: March 31, 2014)

\begin{abstract}
The recent CMB data from Planck and BICEP2 observations have opened a new window for inflationary cosmology. In this Essay we compare three Starobinsky-like inflationary scenarios: (i) the original Starobinsky proposal; (ii) a family of dynamically broken SUGRA models; and (iii) a class of "decaying" vacuum $\Lambda(H)$ cosmologies. We then focus on the $\Lambda(H)$ variant, which spans the complete cosmic history of the universe from an early inflationary stage, followed by the "graceful exit" into the standard radiation regime, the matter epoch and, finally, the late-time accelerated expansion. Computing the effective potential we find that the "running" $\Lambda(H)$ models also provide a prediction for the tensor-to-scalar ratio of the CMB spectrum, $r \simeq 0.16$, which is compatible to within $1 \sigma$ with the value $r=0.20_{-0.05}^{+0.07}$ recently measured by the BICEP2 collaboration.
\end{abstract}

\footnotetext{
${ }^{1}$ Essay selected with honorable mention from Gravity Research Foundation (2014) - Awards Essays on Gravitation
} 
After decades of the successful big-bang paradigm, cosmology still lacks a framework in which the early inflationary phase of the universe smoothly matches the radiation epoch and evolves to the current quasi-de Sitter stage. Indeed, in the light of the Planck and BICEP2 results [1, 2], a heated debate is taking place in the literature about the best implementation of the inflationary paradigm[3, 4].

Ijjas, Steinhardt and Loeb (ISL, [3] ) claimed that there is a new theoretical puzzle termed the 'unlikeliness problem'. It comes from the fact that only models with plateau potentials are favored by Planck, but such scenarios are generically plagued with problems. For example, the initial smoothness must be much larger than the Hubble volume, and this means that inflation can only begin if the universe is extremely homogeneous. In addition, there is a long debate on the production of multi-universes and their measure [5].

Such criticisms were answered in a recent paper by Guth, Kaiser and Nomura (GKN, [4]). Despite they agreed that some criticisms of the ISL paper are correct (for instance the Multiverse Problem[5]), their basic conclusion is that inflation is a robust scenario which is on 'strong footing than ever'.

The lively debate is going on, but in the meanwhile crucial new data also came to shed some light on it. This is precisely the context of our Essay. Here we focus on some variants of Starobinsky's inflation [6] that turn out to be quite promising in the light of the data by Planck and the hot recent one from BICEP2 [1, 2].

The original Starobinsky Inflation: It is the framework that implements de Sitter (inflationary) cosmological solution of the gravitational field equations including higher curvature terms $\propto R^{2}$ in the effective action [6]:

$$
\mathcal{S}=\frac{1}{2 \kappa^{2}} \int d^{4} x \sqrt{-g}\left(R+\beta R^{2}\right), \beta=\frac{8 \pi}{3 \mathcal{M}^{2}},
$$

where $R$ is the Ricci scalar, $\kappa^{2}=8 \pi G$ and $G=1 / M_{P}^{2}$ is Newton's (gravitational) constant in four space-time dimensions, with $M_{P}$ the Planck mass, and $\mathcal{M}$ a characteristic mass scale of the model. The important feature of this model is that inflationary dynamics is driven by the purely gravitational sector, through the $R^{2}$ terms, and the scale of inflation is linked to $\mathcal{M}$. However, the manifest disagreement of the BICEP2 measurement [2] of the tensorto-scalar ratio $r \simeq 0.2$ with the very small value $r=12 / N^{2}(N \simeq 50$ being the number of e-folds) predicted by the original Starobinsky model, has triggered new formulations. 
Dynamically Broken SUGRA: The compatibility of the dynamical breaking of supergravity (SUGRA) theories via gravitino condensation with Starobinsky [6] inflationary scenario was also recently discussed [7]. Dynamical breaking of SUGRA, in the sense of the generation of a mass for the gravitino field, whilst the gravitons remain massless, occurs in the model as a result of the four-gravitino interactions. The one-loop effective potential, obtained by integrating out gravitons and (massive) gravitino fields in the scalar channel, may be expressed as a power series in $\Lambda$. The terms of order $\Lambda^{2}$ combine into curvature scalar square terms, and the effective action reads

$$
S_{S U G R A} \simeq \frac{1}{2 \kappa^{2}} \int d^{4} x \sqrt{-g}\left(\alpha_{1} \widehat{R}+\alpha_{2} \widehat{R}^{2}\right)
$$

with $\widehat{R}$ denoting the fixed $S^{4}$ background one expands around $\left(\widehat{R}=4 \Lambda\right.$, Volume $\left.=24 \pi^{2} / \Lambda^{2}\right)$, and the $\alpha$ 's indicate the graviton and gravitino quantum corrections at each order in $\Lambda$. Obviously, one can see a link between the action (2) with a Starobinsky type action (11), through an effective scale $\beta_{\text {eff }} \equiv \alpha_{2} / \alpha_{1}$.

Running Vacuum: It is remarkable that the Cosmological Principle (embodied in the FLRW metric) does not prevent $\Lambda$ to evolve with cosmic time or a function of it. Thus, in the Einstein equations, $R_{\mu \nu}-(1 / 2) g_{\mu \nu} R+\Lambda g_{\mu \nu}=-8 \pi G T_{\mu \nu}$, the $\Lambda$-term can vary with time on equal footing to the matter density or the scale factor of the FLRW metric. In fact the suggested time evolution of $\Lambda$ is indirect through the Hubble function $H(t)$. Specifically, the quantum field theory (QFT) in curved space-time, in combination with the renormalization group (RG), singles out the possible general form of the evolution of the vacuum energy density, $\rho_{\Lambda}=\Lambda /(8 \pi G)$, as a function of $H(t)$ - acting here as the natural running scale of the cosmic evolution. It suggests a RG-equation in which the rate of change of $\rho_{\Lambda}$ with $H(t)$ contains only even powers of $H$ (because of the covariance of the effective action) [8]:

$$
\frac{d \rho_{\Lambda}}{d \ln H^{2}}=\frac{1}{(4 \pi)^{2}} \sum_{i}\left[a_{i} M_{i}^{2} H^{2}+b_{i} H^{4}+c_{i} \frac{H^{6}}{M_{i}^{2}}+\ldots\right],
$$

where the (dimensionless) coefficients receive loop contributions from boson and fermion (hereafter $b$ and $f$ ) matter fields of different masses $M_{i}$. There are no $M_{i}^{4}$ terms on the r.h.s. of (3) as $H<M_{i}$ for all particles and hence the RG prevents those terms from appearing [8]. Obviously the expansion converges very fast at low energies, where $H$ is rather small certainly much smaller than any particle mass. No other term beyond $H^{2}$ (not even $H^{4}$ ) can 
contribute significantly on the r.h.s. of equation (3) at any stage of the cosmological history below the GUT scale $M_{G U T}$, typically a few orders of magnitude below the Planck scale $M_{P} \sim 10^{19} \mathrm{GeV}$. In contrast, in the very early universe (when $H$ is close, but below, the masses of the heavy fields $M_{i} \sim M_{G U T}$ ), the $H^{4}$ effects can be significant, in fact dominant. Integrating the above equation with only one leading high power term of $H$, generically called $H^{n+2}(n \geq 1)$, we arrive at

$$
\Lambda(H)=c_{0}+3 \nu H^{2}+3 \alpha \frac{H^{n+2}}{H_{I}^{n}} .
$$

Here $c_{0}$ is an integration constant and $H_{I}$ is the Hubble parameter at the inflationary scale generated by the $H^{n+2}$ term. The coefficients read: $\nu=\frac{1}{6 \pi} \sum_{i=f, b} c_{i} \frac{M_{i}^{2}}{M_{P}^{2}}$, $\alpha=\frac{1}{12 \pi} \frac{H_{I}^{2}}{M_{P}^{2}} \sum_{i=f, b} b_{i}$. They receive contributions from all the matter particles and play the role of one-loop $\beta$-functions for the RG running. Both coefficients are predicted to be naturally small since $M_{i}^{2} \ll M_{P}^{2}$ for all the particles, even for the heavy fields of a typical GUT below the Planck scale). In the case of $\nu$ an estimate within a generic GUT is found in the range $|\nu|=10^{-6}-10^{-3}[9]$, compatible with observations [10].

The equations of state for the dynamical vacuum and matter fluids are still $p_{\Lambda}(t)=-\rho_{\Lambda}(t)$ and $p=\omega \rho$ ( $\omega$ constant $)$, respectively. The overall conservation law in the presence of a dynamical $\Lambda$-term reads $\dot{\rho}+3(1+\omega) H \rho=-\dot{\rho_{\Lambda}}$, entailing energy exchange between matter and vacuum. Combining this conservation equation with (4) and Friedmann's equation in flat space, $8 \pi G\left(\rho+\rho_{\Lambda}\right)=3 H^{2}$, we obtain the differential evolution law for $H(t)$. Let us express these results for $n=2\left(H^{4}\right.$-inflation $)$, which is the basic model. We find:

$$
\dot{H}+\frac{3}{2}(1+\omega) H^{2}\left[1-\nu-\frac{c_{0}}{3 H^{2}}-\alpha\left(\frac{H}{H_{I}}\right)^{2}\right]=0 .
$$

It admits the constant solution $H=H_{I}[(1-\nu) / \alpha]^{1 / 2}$, corresponding to an inflationary regime in the very early universe, i.e. when $H^{2} \gg c_{0}$. The solution of Eq.(5) is completely analytical, it suffices to say that $H(a) / H_{I} \propto\left[1+D a^{4(1-\nu)}\right]^{-1 / 2}$ in the radiation epoch $(\omega=1 / 3)$, where $D>0$ is a constant of integration. For $D a^{4(1-\nu)} \ll 1$ the universe starts without a singularity from the mentioned inflationary phase powered by the huge value $H_{I}$, presumably connected to the scale of a Grand Unified Theory (GUT) or even the Planck scale $M_{P}$. At this epoch we have $\Lambda(H) \simeq 3 \alpha H^{4} / H_{I}^{2}$ and $\dot{H}=0$, whereas the Ricci scalar becomes $R=12 H^{2}$. From the foregoing we may roughly expect that the pure gravitational 
part of the underlying effective action should behave approximately as

$$
S_{R, \Lambda} \simeq \frac{1}{2 \kappa^{2}} \int d^{4} x \sqrt{-g}\left(R+\tilde{\beta} R^{2}\right), \quad \tilde{\beta}=-\frac{\alpha}{24 H_{I}^{2}}
$$

Here $\tilde{\beta}$ plays the role of an effective Starobinsky coefficient: for $\tilde{\beta} \equiv \beta$ the time varying vacuum model (6) can "effectively" be compared to the Starobinsky inflationary model (11), while for $\tilde{\beta} \equiv \beta_{\text {eff }}$ it bares relation with dynamically broken SUGRA inflationary scenario (2) [11]. Of course there is no equivalence among these models, but they share some similarities.

The differences can actually be significant and may prove advantageous in some cases. In fact, our main question in this work is the following: what about the truly distinctive bonuses of the $\Lambda(H)$ class? There are quite a few.

Firstly, for $D a^{4(1-\nu)} \gg 1$ the universe evolves smoothly into essentially the standard radiation phase $H(a) \simeq a^{-2(1-\nu)}(|\nu| \ll 1)$ and therefore successfully implements the "graceful exit" from the de Sitter stage. Then, as the expansion proceeds, the radiation component becomes sub-dominant, giving rise to the matter-dominated era. At this point the $H^{n+2}$-term in (4) becomes negligible. Assuming flat 3-dimensional space and integrating Eq.(5) we obtain $H^{2}(a) / H_{0}^{2}=\left[\left(1-\Omega_{\Lambda}^{0}\right) a^{-3(1-\nu)}+\Omega_{\Lambda}^{0}-\nu\right] /(1-\nu)$. This low-energy model turns out to be in agreement with the latest cosmological data, including the growth rate of clustering basically as in the $\Lambda \mathrm{CDM}$ [10,12]. Not only so, a complete cosmological description emerges where the universe evolves between two extreme (early and late-time) de Sitter accelerating stages, with the ratio of the corresponding densities given by $\rho_{f} / \rho_{I} \sim\left(H_{0} / H_{I}\right)^{2} \sim 10^{-120}$ (for $H_{I}^{-1}$ close but below the Planck time). See e.g. the detailed discussion in [12], as well as a plot of the complete cosmic history in Fig. 1 of [13].

Finally, we compute the tensor-to-scalar ratio $(r)$ and the spectral index $\left(n_{s}\right)$ encoded in the CMB map within the effective potential approximation associated to the model (44) [13]. The potential can be expressed as follows:

$$
\frac{V_{\mathrm{eff}}(\phi)}{V(0)}=\frac{1+(1 / 3) \sinh ^{2}(n \phi / 2)}{\left[1+\sinh ^{2}(n \phi / 2)\right]^{(n+2) / n}} .
$$

The standard slow-roll parameters $(\epsilon, \eta)$ can now be readily calculated, and from them $\left(n_{s}, r\right)$ immediately ensue. To this effect we perform a Taylor expansion of the effective potential around $\phi=0$, keeping terms up to $\phi^{2}$ and using the fact that $V^{\prime \prime}(0) / V(0)=-n(n+3) / 3$. 
The final result for the CMB parameters within this approximation reads:

$$
r=16 \epsilon \simeq 32 N\left[\frac{n(n+3)}{f_{N}-3}\right]^{2}, \quad n_{s}-1=-6 \epsilon+2 \eta \simeq-\frac{2 n(n+3)\left(2 f_{N}+3\right)}{\left(f_{N}-3\right)^{2}},
$$

where $f_{N} \equiv 2 N n(n+3)$, with $N$ the number of e-folds. Using $N \simeq 50$ and $n=2$ (corresponding to $H^{4}$-inflation, which is the minimal model compatible with general covariance of the effective action) we obtain $r \simeq 0.161$. This result is consistent with the value $r=0.20_{-0.05}^{+0.07}$ that has been recently measured by the BICEP2 collaboration [2].

Within this approach we find an universal prediction that is valid for virtually all $H^{n+2}$ models $(n \geq 1)$, to wit: $r \rightarrow r^{u} \simeq 8 / N \simeq 0.16$ and $n_{s} \rightarrow n_{s}^{u} \simeq 1-2 / N \simeq 0.96$ (for $N=50$ ), a result which is in agreement with the $1 \sigma$ BICEP2 measurements [2].

We remark that $n_{s}^{u}$ is exactly as in the original Starobinsky's model. However, the predicted $r$ in that model is too small, $r=12 / N^{2} \simeq 4.80 \times 10^{-3}$ for $N=50$. Our value of $r^{u}$ is $2 N / 3 \gtrsim 33$ times bigger and can be in better agreement with observation.

To summarize, the inflationary class $H^{n+2}$ of models (44) seems to accomplish three main achievements $\forall n \geq 1$ : i) it provides "graceful exit"; ii) it smoothly connects the early universe with the $\Lambda \mathrm{CDM}$ model; and iii) it leads to a prediction compatible with the CMB parameters $n_{s}$ and $r$ as measured by the combined Planck, WMAP, BAO and BICEP2 data: $n_{s}=0.9607 \pm 0.0063$ and $r=0.20_{-0.05}^{+0.07}[1,2]$.

[1] P. A. R. Ade et al. [Planck Collaboration] arXiv:1303.5082]; arXiv:1303.5062].

[2] BICEP2 Collaboration (P. A. R. Ade (Cardiff U.) et al.) arXiv:1403.3985].

[3] A. Ijjas, P. J. Steinhardt and A. Loeb, Phys. Lett. B. 723, 261 (2013), and arXiv:1402.6980.

[4] A. H. Guth, D. I. Kaiser and Y. Nomura arXiv:1312.7619]; cf also A. Linde arXiv:1402.0526].

[5] The continued multiverse production implies that "anything that can happen will happen an infinite number of times". See A. Guth, J. Phys. A 40, 6811 (2007).

[6] A. A. Starobinsky, Phys. Lett. B. 91, 99 (1980).

[7] J. Alexandre, N. Houston and N. E. Mavromatos, Phys. Rev. D 89, 027703 (2014).

[8] I. L. Shapiro and J. Solà, JHEP 02, 006 (2002); Phys. Lett. B 475, 236 (2000); Nucl. Phys. Proc. Suppl. 127, 71 (2004). 
[9] J. Solà, J. of Phys. A. 41, 164066 (2008).

[10] S. Basilakos, M. Plionis, and J. Solà, Phys. Rev. D 80, 083511 (2009).

[11] J. Ellis and N. E. Mavromatos, Phys. Rev. D. 88, 085029 (2013).

[12] J. A. S. Lima, S. Basilakos and J. Solà, Mon. Not. R. Astron. Soc. 431, 923 (2013); E. L. D. Perico, J. A. S. Lima, S. Basilakos and J. Solà, Phys. Rev. D 88, 063531 (2013).

[13] S. Basilakos, J. A. S. Lima, and J. Solà, Int. J. Mod. Phys. D 22, 1342008 (2013). 